\title{
ON THE RESIDUES OF A CUBIC POLYNOMIAL (Mod p)
}

\author{
K. McCann and K.S. Williams
}

(received November 18, 1966)

If $f(x)$ is a polynomial with integral coefficients then the integer $r$ is said to be a residue of $f(x)$ modulo an integer $m$ if the congruence

$$
f(x) \equiv \mathbf{r}(\bmod m)
$$

is soluble for $x$; otherwise $r$ is termed a non-residue. When $m$ is a prime $p$, Mordell [4] has shown that the least nonnegative residue $\ell$ of $f(x)(\bmod p)$ satisfies

$$
\ell \leq \mathrm{d} \mathrm{p} \mathrm{p}^{1 / 2} \log \mathrm{p} \text {, }
$$

where $d$ is the degree of $f(x)$. When $f(x)$ is a cubic he has also shown that the least non-negative non-residue $k$ of $f(x)$ $(\bmod p)$ is ${ }^{*} 0\left(p^{1 / 2} \log p\right)$. It is the purpose of this note to discuss the distribution of the residues of the cubic $f(x)(\bmod p)$ in greater detail. To keep the notation simple we take $f(x)$ in the. form $x^{3}+a x ;$ no real loss of generality is involved, everything we do for $\mathrm{x}^{3}+\mathrm{ax}$ can be done for $\mathrm{Ax^{3 }}+\mathrm{Bx^{2 }}+\mathrm{Cx}+\mathrm{D}$ but at the cost of complicating the notation. When $a \equiv 0(\bmod p)$, $f(x)=x^{3}$ and our results are well-known in this case. Henceforth we assume that a $\neq 0(\bmod p)$. Let

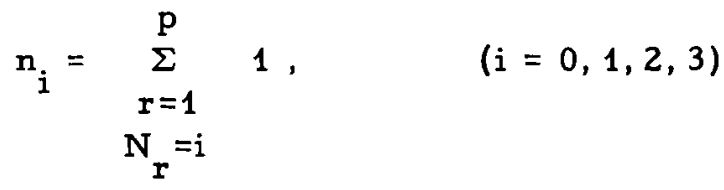

Unless otherwise stated all constants implied by 0 -symbols are absolute.

Canad. Math. Bull. vol. 10, no. 1, 1967 
where $\mathrm{N}_{\mathbf{r}}$ denotes the number of solutions $\mathrm{x}$ of

$$
x^{3}+a x \equiv r(\bmod p) \text {. }
$$

It is well-known that for $p>3$

$$
n_{1}=\frac{1}{2}\left\{p+\left(\frac{-3}{p}\right)-\left(\frac{-3 a}{p}\right)-1\right\} \text {, }
$$

$$
n_{2}=\left(\frac{-3 a}{p}\right)+1
$$

and

$$
n_{3}=\frac{1}{6}\left\{p-\left(\frac{-3}{p}\right)-3\left(\frac{-3 a}{p}\right)-3\right\} .
$$

Hence the number of residues of $x^{3}+\operatorname{ax}(\bmod p)$, which is just $n_{1}+n_{2}+n_{3}$, is

$$
\frac{1}{3}\left\{2 p+\left(\frac{-3}{p}\right)\right\}=\frac{2}{3} p+0(1)
$$
as $\mathrm{p} \rightarrow \infty$.

This tells us that, for large $p$, approximately two-thirds of the integers

$$
1,2,3, \ldots, p
$$

are residues of $x^{3}+a x$. We show that this is also true for

$$
1,2,3, \ldots, \mathrm{h}
$$

provided $h$ is sufficiently large. More precisely we show that the number of residues of $x^{3}+$ ax in (8) is

$$
\frac{2}{3} h+O\left(p^{1 / 2} \log p\right)
$$

A consequence of this is Mordell's estimate for $k$. In addition, as $\frac{2}{3}>\frac{1}{2}$, it shows that the least pair of consecutive positive 
residues is also $0\left(p^{1 / 2} \log p\right)$.

In the proof of (9) (and later) we use Vinogradov's method for incomplete character and exponential sums. This requires the familiar Polya-Vinogradov inequality, namely,

$$
\underset{y=1}{p-1}\left|\sum_{x=1}^{h} e(y x)\right| \leq p \log p
$$

for $p \geq 61$, where $e(t)$ denotes $\exp \left(2 \pi i t p^{-1}\right)$. For the complete sums involved we appeal to the general estimates of Perel'muter [5]. These include the estimate of Carlitz and Uchiyama [2], used by Mordell in [4], namely

$$
\left|\sum_{x=1}^{p} e(f(x))\right| \leq(d-1) p^{1 / 2}
$$

where $d$ denotes the degree of the polynomial $f$, and Weil's estimate [6] for the Kloosterman sum, i.e.,

$$
\left|\sum_{x=1}^{p-1} e\left(a x+b x^{-1}\right)\right| \leq 2 p^{1 / 2}
$$

where $x^{-1}$ denotes the inverse of $x(\bmod p)$ and $a, b \neq 0$ $(\bmod p)$. All these estimates are consequences of Weil's proof of the Riemann hypothesis for algebraic function fields over a finite field.

Analogous to (1) we set

$$
\mathrm{m}_{\mathrm{i}}=\sum_{\substack{\mathrm{r}=1 \\ \mathrm{~N}_{\mathbf{r}}=\mathrm{i}}}^{\mathrm{h}} 1 \quad(\mathrm{i}=0,1,2,3)
$$

so that we require $m_{1}+m_{2}+m_{3}$. From [4] we have

$$
m_{2}=0(1)
$$

and from Mordell's paper [4] 


$$
m_{1}+2 m_{2}+3 m_{3}=h+0\left(p^{1 / 2} \log p\right)
$$

so that it suffices to determine $m_{1}$. Now (2) has one solution if and only if

$$
\left(\frac{-4 a^{3}-27 r^{2}}{p}\right)=-1
$$

so

$$
m_{1}=\frac{1}{2} \sum_{r=1}^{h}\left\{1-\left(\frac{-4 a^{3}-27 r^{2}}{p}\right)\right\}+0(1) .
$$

Applying Vinogradov's method and appealing to Perel'muter's results [5] (or to Weil's estimate (12) for the Kloosterman sum) we have

$$
\sum_{r=1}^{h}\left(\frac{-4 a^{3}-27 r^{2}}{p}\right)=0\left(p^{1 / 2} \log p\right)
$$

so that

$$
m_{1}=\frac{1}{2} h+0\left(p^{1 / 2} \log p\right)
$$

We now consider pairs of consecutive residues of $x^{3}+\operatorname{ax}(\bmod p)$. Define $n_{i j}(0 \leq i, j \leq 3)$ by

$$
n_{i j}=\sum_{r=1}^{p} 1
$$

so that the number of such pairs is just

$$
\sum_{1 \leq i, j \leq 3}^{\sum} n_{i j}
$$

From (4) $n_{i 2}, n_{2 j}=0(1)$ for $0 \leq i, j \leq 3$. Also it is easy to 
show that $n_{13}=n_{31}$ so it suffices to evaluate $n_{11}, n_{13}$ and $\mathrm{n}_{33}$. We begin by showing that

$$
\mathrm{n}_{11}=\frac{\mathrm{p}}{4}+0\left(\mathrm{p}^{1 / 2}\right) \text {. }
$$

We have

$$
\begin{aligned}
& n_{11}=\sum_{r=1}^{p} 1 \\
& \left(\frac{-4 a^{3}-27 r^{2}}{p}\right)=-1,\left(\frac{-4 a^{3}-27(r+1)^{2}}{p}\right)=-1 \\
& =\frac{1}{4} \sum_{r=1}^{p}\left\{1-\left(\frac{-4 a^{3}-27 r^{2}}{p}\right)\right\}\left\{1-\left(\frac{-4 a^{3}-27(r+1)^{2}}{p}\right)\right\}+0(1)
\end{aligned}
$$

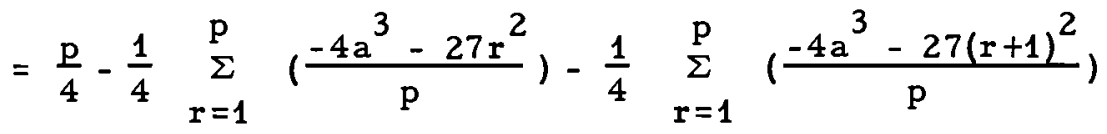

$$
\begin{aligned}
& +\frac{1}{4} \sum_{r=1}^{p}\left(\frac{\left(-4 a^{3}-27 r^{2}\right)\left(-4 a^{3}-27(r+1)^{2}\right)}{p}\right)+0(1) .
\end{aligned}
$$

The first two character sums are $0(1)$ and the last one by Perel'muter's results is $\leq 3 \mathrm{p}^{1 / 2}$ in absolute value, since $\left(-4 a^{3}-27 r^{2}\right)\left(-4 a^{3}-27(r+1)^{2}\right)$ is not identically $(\bmod p)$ a square in $r$.

We next prove that

$$
\mathrm{n}_{13}=\frac{\mathrm{p}}{12}+0\left(\mathrm{p}^{1 / 2}\right) \text {. }
$$

We do this by showing that

$$
n_{11}+2 n_{12}+3 n_{13}=\frac{p}{2}+0\left(p^{1 / 2}\right) \text {. }
$$

(20) follows since we know $n_{11}$ and $n_{12}$. We have 


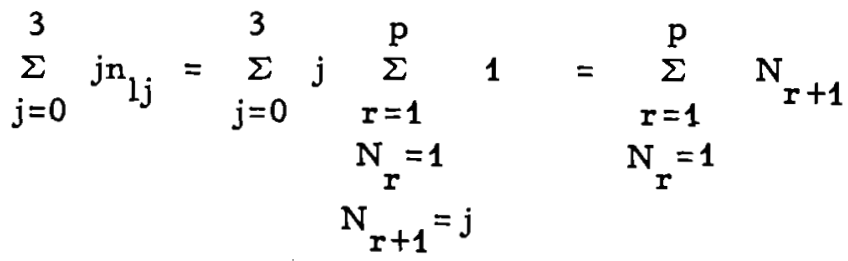

$$
\begin{aligned}
& =\begin{array}{cc}
\sum_{\mathrm{r}=1}^{\mathrm{p}} & \mathrm{p} \\
\left(\frac{-4 \mathrm{a}^{3}-27 \mathrm{r}^{2}}{\mathrm{p}}\right)=-1 & \mathrm{x}^{3}+\mathrm{ax} \equiv \mathrm{x}+1
\end{array} \\
& =\frac{1}{2} \sum_{x=1}^{p}\left\{1-\left(\frac{-4 a^{3}-27\left(x^{3}+a x-1\right)^{2}}{p}\right)\right\}+0(1) \\
& =\frac{p}{2}-\frac{1}{2} \sum_{x=1}^{p}\left(\frac{-4 a^{3}-27\left(x^{3}+a x-1\right)^{2}}{p}\right)+0(1) .
\end{aligned}
$$

Now $27^{2}\left(x^{3}+a x-1\right)^{2}+108 a^{3}$ is not identically (mod p) a square in $x$ as a $\neq 0(\bmod p)$. Hence Perel'muter's work tells us that the character sum is $0\left(\mathrm{p}^{1 / 2}\right)$. This proves $(21)$.

Finally consider

$$
\mathrm{n}_{11}+2\left(\mathrm{n}_{12}+\mathrm{n}_{21}\right)+3\left(\mathrm{n}_{13}+\mathrm{n}_{31}\right)+4 \mathrm{n}_{22}+6\left(\mathrm{n}_{23}+\mathrm{n}_{32}\right)+9 \mathrm{n}_{33} \text {. }
$$

This is just the number of solutions $(x, y)$ of

$$
\left(x^{3}+a x\right)-\left(y^{3}+a y\right)-1 \equiv 0(\bmod p) .
$$

By a result of Lang and Weil [3] this number is

$$
p+0\left(p^{1 / 2}\right)
$$

Hence

$$
n_{33}=\frac{p}{36}+0\left(p^{1 / 2}\right) .
$$


Thus the number of pairs of consecutive residues is

$$
\frac{4}{9} p+0\left(p^{1 / 2}\right)
$$

We conclude by calculating the number of pairs of residues of $x^{3}+\operatorname{ax}(\bmod p)$ in $(8)$. We define $m_{i j}(0 \leq i, j \leq 3)$ by

$$
m_{i j}=\sum_{r=1}^{p} 1 .
$$

From (4) we have $m_{i 2}, m_{2 j}=0(1)(0 \leq i, j \leq 3)$ and, much as before, we can show that

$$
m_{11}=\frac{h}{4}+0\left(p^{1 / 2} \log p\right)
$$

and

$$
m_{13}=m_{31}=\frac{h}{12}+0\left(p^{1 / 2} \log p\right) .
$$

The only difficulty is the estimation of $\mathrm{m}_{33}$. We find it necessary to appeal to a recent deep estimate of Bombieri and Davenport [1] for an exponential sum of the type

$$
\begin{gathered}
\stackrel{p}{\Sigma} e(f(x)) \\
x, y=1 \\
q(x, y) \stackrel{\equiv}{\equiv} 0(\bmod p)
\end{gathered}
$$

where $\phi(x, y)$ is absolutely irreducible $(\bmod p)$. We have

$$
\begin{gathered}
m_{11}+2\left(m_{12}+m_{21}\right)+3\left(m_{13}+m_{31}\right)+4 m_{22}+6\left(m_{23}+m_{32}\right)+9 m_{33} \\
=\sum_{r=1}^{h} N_{r} N_{r+1}
\end{gathered}
$$




$$
\begin{aligned}
& =\frac{1}{p} \underset{r=1}{\sum} \underset{s=1}{i} \underset{t=1}{\mathrm{~h}} \mathrm{~N}_{r} \mathrm{~N}_{r+1} \mathrm{e}(\mathrm{t}(\mathrm{r}-\mathrm{s})) \\
& =\frac{h}{p} \underset{r=1}{p} N_{r} N_{r+1}+\frac{1}{p} \underset{t=1}{p-1}\left\{\underset{r=1}{p} N_{r} N_{r+1} e(t r)\right\}\left\{\underset{s=1}{\sum} e(-s t)\right\} .
\end{aligned}
$$

\section{Hence}

$$
\begin{aligned}
& \left|m_{11}+2\left(m_{12}+m_{21}\right)+\ldots+9 m_{33}-\frac{h}{p}\left(p+o\left(p^{1 / 2}\right)\right)\right| \\
& \leq \max _{1 \leq t \leq p-1}\left|\sum_{r=1}^{p} N_{r} N_{r+1} e(t r)\right| \log p .
\end{aligned}
$$

Now

$$
\sum_{r=1}^{p} N_{r} N_{r+1} e(t r)
$$

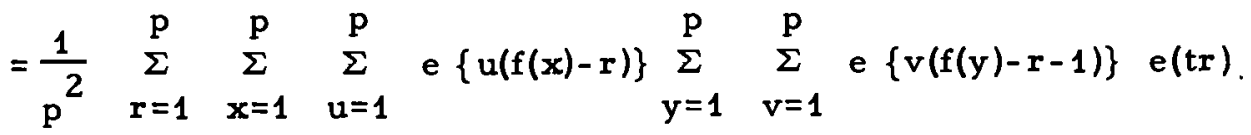

$=\frac{1}{p^{2}} \underset{x, y, u, v=1}{\stackrel{p}{\Sigma} e}\{u f(x)+v f(y)-v\} \underset{r=1}{p} e\{(t-u-v) r\}$

$=\frac{1}{p} \underset{x, y, v=1}{p}$ e $\{(t-v) f(x)+v f(y)-v\}$

$=\frac{1}{p} \sum_{x, y=1}^{p} e\{t f(x)\} \sum_{y=1}^{p} e\{v(f(y)-f(x)-1)\}$

$=\quad \sum^{p} \quad e(t f(x))$.

$x, y=1$

$f(y)-f(x)-1 \equiv 0$ 
As $f(y)-f(x)-1$ is absolutely irreducible $(\bmod p)$, by the mentioned result of Davenport and Bombieri, this sum in absolute value is less than $18 \mathrm{p}^{1 / 2}+9$. Hence

$$
m_{33}=\frac{h}{36}+0\left(p^{1 / 2} \log p\right)
$$

and the number of pairs of consecutive residues in (8) is

$$
\frac{4 h}{9}+0\left(p^{1 / 2} \log p\right) \text {. }
$$

This implies that the least triple of consecutive positive residues of $x^{3}+a x(\bmod p)$ is also $0\left(p^{1 / 2} \log p\right)$.

In conclusion we would like to say that a number of modifications of this work are possible; for example the results obtained can be extended to arbitrary arithmetic progressions without difficulty and also to quartic polynomials. Finally we offer the following

CONJECTURE: For a fixed positive integer $k$ the number $N_{k}(a)$ of blocks of $k$ consecutive residues of $x^{3}+\operatorname{ax}(\bmod p)$ satisfies

$$
\lim _{p \rightarrow \infty} \frac{N_{k}(a)}{p}=\left(\frac{2}{3}\right)^{k}
$$

for each $k$, uniformly in a $\neq 0(\bmod p)$.

This has been verified for $k=1$ and 2 .

\section{REFERENCES}

1. E. Bombieri and H. Davenport, On Two Problems of Mordell. Amer. Jour. Math., 88 (1966), pages 61-70.

2. L. Carlitz and S. Uchiyama, Bounds for Exponential Sums. Duke Math. Jour., 24 (1957), pages 37-41.

3. S. Lang and A. Weil, Number of Points of Varieties in Finite Fields. Amer. Jour. Math., 76 (1954), pages 819-827. 
4. L. J. Mordell, On the Least Residue and Non-residue of a Polynomial. Jour. Lond. Math. Soc., 38 (1963), pages 451-453.

5. G.I. Perel'muter, On Certain Sums of Characters. Uspektii Matematicheskikh Nauk., 18 (1963), pages 145-149.

6. A. Weil, On Some Exponential Sums. Proc. Nat. Acad. Sci. (U.S.A.), 34 (1948), pages 204-207.

Manchester University

Manchester, England

Carleton Univer sity

Ottawa, Canada 